\title{
Richtlinien «Patientenverfügungen» definitiv verabschiedet
}

Peter Lack, Michelle Salathé

Der Senat der SAMW hat die Richtlinien «Patientenverfügungen» am 19. Mai 2009 verabschiedet. Die definitive Version der Richtlinien kann von der Website der SAMW (www.samw.ch) heruntergeladen oder in Broschüreform beim Generalsekretariat der SAMW, Petersplatz 13, $4051 \mathrm{Ba-}$ sel, bestellt werden.

Korrespondenz:

Lic. iur. Michelle Salathé

Generalsekretariat der SAMW

Petersplatz 13

CH-4051 Basel
Im November 2008 hat die Schweizerische Akademie der Medizinischen Wissenschaften (SAMW) Richtlinien zum Erstellen von Patientenverfügungen und deren Umsetzung im medizinischen Alltag zur Vernehmlassung veröffentlicht. Mit rund 80 eingegangenen Stellungnahmen war das Echo auf das Dokument gross und grösstenteils positiv. Der Richtlinien-Entwurf wurde als «wertvoller, praxisbezogener Beitrag zum Thema Patientenverfügung» geschätzt. Im Zentrum der kritischeren Rückmeldungen stand der Wunsch nach einer klaren Aussage bezüglich der Verbindlichkeit der Patientenverfügung sowie der Bedeutung der Beratung und Aufklärung beim Verfassen. Die Rückmeldungen zeigten auch bekannte und in der Subkommission diskutierte Spannungsfelder: Einerseits wurde das Bedürfnis nach einer einfachen und «schnellen» Patientenverfügung geäussert, andererseits wurde darauf hingewiesen, dass gerade dies bei der Umsetzung oftmals Schwierigkeiten bereitet. Weiter wurde gewünscht, dass im Adressatenkreis auch Einzelpersonen und Beratungsorganisationen aufgenommen werden.

Die Subkommission unter dem Vorsitz von lic. theol. Peter Lack aus Basel hat diese Anliegen bei der Überarbeitung der Richtlinien berücksichtigt. Bezüglich der Verbindlichkeit orientieren sich die Richtlinien am revidierten Zivilgesetzbuch (Erwachsenenschutz), welches frühestens 2012 in Kraft tritt. Bis zu diesem Zeitpunkt gelten die auf kantonaler Ebene bestehenden rechtlichen Regelungen zur Patientenverfügung. Diese variieren allerdings stark und einige Kantone haben keine explizite Regelung. Trotzdem gilt bereits heute: Je klarer eine Patientenverfügung ist und je konkreter sie auf die aktuelle medizinische Entscheidungssituation zutrifft, desto gewichtiger ist ihre Rolle.

\section{Inhalte einer Patientenverfügung}

In einer Patientenverfügung kann eine Person festhalten, welchen medizinischen Massnahmen sie zustimmt und welche sie ablehnt. Sie kann sich zu weiteren Themen wie z.B. Obduktion, Transplantation usw. äussern und/oder eine Vertretungsperson bezeichnen. Weil im Voraus oft nur schwer abschätzbar ist, welche medizinischen Massnahmen in der konkreten Situation angezeigt sind, empfehlen die Richtlinien den Verfassenden, die persönliche Werthaltung zu umschreiben. Angaben zur Werthaltung können Orientierung bieten, wenn sich der Verfügende nicht explizit zu bestimmten Situationen oder Massnahmen geäussert hat oder nicht absehbar ist, ob eine medizinische Massnahme den gewünschten Erfolg bringt.
In einer Patientenverfügung können keine Handlungen gefordert werden, die mit dem Recht nicht vereinbar sind. Es können auch keine Behandlungen eingefordert werden, die medizinisch nicht indiziert sind. Hingegen können Behandlungen, die medizinisch indiziert wären, abgelehnt werden. Damit keine Zweifel bezüglich der Informiertheit der Willensbildung aufkommen, sollten in diesem Fall auch die Beweggründe, die zu dieser Ablehnung führen, in der Patientenverfügung zur Sprache kommen.

\section{Beratung empfehlenswert, aber kein Muss}

Die Richtlinien halten ausdrücklich fest, dass keine Verpflichtung besteht, sich beim Erstellen einer Patientenverfügung beraten zu lassen; aus Sicht der SAMW ist ein Beratungsgespräch aber hilfreich und zu empfehlen. Eine solche Beratung kann vom Hausarzt, dem Facharzt oder einer mit der Thematik vertrauten Person übernommen werden. Die Beratung bietet insbesondere die Chance, dass die Verfügende bei der Reflexion und Dokumentation der persönlichen Werthaltung unterstützt und über mögliche Situationen der Urteilsunfähigkeit sowie über die üblicherweise vorgesehenen medizinischen Massnahmen informiert wird. Im Beratungsgespräch sollte der Verfügende über die Konsequenzen der Einleitung bzw. des Verzichts oder Abbruchs von Massnahmen aufgeklärt werden.

\section{Umsetzung der Patientenverfügung}

Die Richtlinien äussern sich auch zur Situation, in welcher eine Patientenverfügung möglicherweise nicht mehr dem Willen des Patienten entspricht, das Dokument aber nicht widerrufen oder geändert wurde. Beschrieben sind auch Situationen, in welchen sich die behandelnden Ärztinnen und Ärzte, Pflegefachpersonen, Vertretungspersonen und Angehörige uneinig bezüglich der Auslegung einer Patientenverfügung sind. Für diese Fälle wird in den Richtlinien festgehalten, wie eine Entscheidungsfindung geschehen sollte und welche unterstützenden Massnahmen ergriffen werden können.

Den in der Vernehmlassung geäusserten Wünschen nach einer kurzen Patientenverfügung einerseits und dem Bedürfnis, die Aussagekraft von Patientenverfügungen für die konkrete Umsetzungssituation zu fördern, andererseits, trägt die SAMW Rechnung. In Vorbereitung sind sowohl eine Kurzfassung der «Richtlinien» als auch Musterfragen für die Beschreibung der Werthaltung. 